\title{
Top-quark effects in diphoton production through gluon fusion at next-to-leading order in QCD
}

\author{
Fabio Maltoni, ${ }^{1,2, *}$ Manoj K. Mandal, ${ }^{3, \dagger}$ and Xiaoran Zhao $\circledast^{1, \$}$ \\ ${ }^{1}$ Centre for Cosmology, Particle Physics and Phenomenology (CP3), Université catholique de Louvain, \\ 1348 Louvain-la-Neuve, Belgium \\ ${ }^{2}$ Dipartimento di Fisica e Astronomia, Università di Bologna and INFN, \\ Sezione di Bologna, via Irnerio 46, 40126 Bologna, Italy \\ ${ }^{3}$ Dipartimento di Fisica e Astronomia, Università di Padova and INFN, \\ Sezione di Padova, Via Marzolo 8, 35131 Padova, Italy
}

(Received 11 January 2019; published 11 October 2019)

\begin{abstract}
At hadron colliders, the leading production mechanism for a pair of photons is from quark-antiquark annihilation at the tree level. However, due to large gluon-gluon luminosity, the loop-induced process $g g \rightarrow \gamma \gamma$ provides a substantial contribution. In particular, the amplitudes mediated by the top quark become important at the $t \bar{t}$ threshold and above. In this paper we present the first complete computation of the next-to-leading order (NLO) corrections (up to $\alpha_{S}^{3}$ ) to this process, including contributions from the top quark. These entail two-loop diagrams with massive propagators whose analytic expressions are unknown and have been evaluated numerically. We find that the NLO corrections to the top-quark induced terms are very large at low diphoton invariant mass $m(\gamma \gamma)$ and close to the $t \bar{t}$ threshold. The full result including five massless quarks and top quark contributions at NLO displays a much more pronounced change of slope in the $m(\gamma \gamma)$ distribution at $t \bar{t}$ threshold than at LO and an enhancement at high invariant mass with respect to the massless calculation.
\end{abstract}

DOI: 10.1103/PhysRevD.100.071501

\section{INTRODUCTION}

The production of a pair of photons (diphoton) is one of the most important processes at hadron colliders. Not only because the final state signature is experimentally very clean, but also because of the great phenomenological relevance for Standard Model (SM) physics and beyond. Its differential cross section has been precisely measured at the Tevatron [1,2] and the LHC [3,4]. The signature has provided one of the two golden channels (the other being $H \rightarrow 4 \ell$ ) for the discovery of the Higgs boson [5,6]. Currently, the $H \rightarrow \gamma \gamma$ decay remains one of the cleanest final states to study the properties of the Higgs boson and its production mechanisms. Being so experimentally neat, the diphoton spectrum is also scrutinized in the search of new physics at the LHC, see e.g., [7,8], such as peak/dip structures coming from new scalar or spin-2 resonances decays and the interference with the standard model background or more exotic features, such as multiple

\footnotetext{
*fabio.maltoni@uclouvain.be, fabio.maltoni@unibo.it †manojkumar.mandal@pd.infn.it

xiaoran.zhao@uclouvain.be
}

Published by the American Physical Society under the terms of the Creative Commons Attribution 4.0 International license. Further distribution of this work must maintain attribution to the author(s) and the published article's title, journal citation, and DOI. Funded by SCOAP. resonances as predicted by extradimensional [9-11] or clockwork models [12].

At hadron colliders, the leading order (LO) contribution to diphoton final states, comes from quark-antiquark annihilation $q \bar{q} \rightarrow \gamma \gamma$. Next-to-leading order (NLO) corrections (at order $\alpha_{S}$ ) to this process have been calculated many years ago [13]. Next-to-next-to-leading order (NNLO) corrections (at order $\alpha_{S}^{2}$ ) have also obtained $[14,15]$ and are available in public codes such as $2 \gamma$ NNLO [14], MCFM [15] and MATRIX [16]. At this order, a new channel arises, i.e., gluons can fuse into diphoton, a quantum process induced by loops of quarks [Fig. 1(a)]. This contribution, while being formally part of the NNLO corrections, is not only finite and gaugeinvariant per se but also anomalously large, due to the gluongluon luminosity. It is common, therefore, to consider loop-induced gluon fusion production which starts at order $\alpha_{S}^{2}$ as an independent diphoton production mechanism. NLO corrections of this process $\left(\alpha_{S}^{3}\right)$, which include two-loop $g g \rightarrow \gamma \gamma$ contributions, were calculated some time ago but only in the case of massless internal quarks $[15,17]$.

The top-quark contribution has been known only at one loop so far. In the low energy region, it is strongly suppressed due to the large top-quark mass, the amplitude scaling as $s^{2} / m_{t}^{4}$. Once the energy becomes comparable to the top mass and in particular close and above the top pair threshold, it becomes enhanced due to the opening of an imaginary part due to rescattering. This transition region is particularly interesting, because it is very sensitive to the 


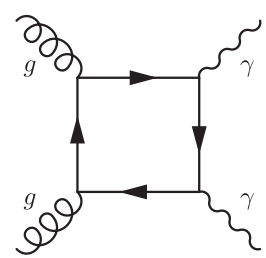

(a)

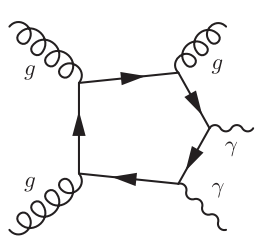

(b)

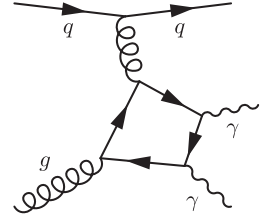

(c)

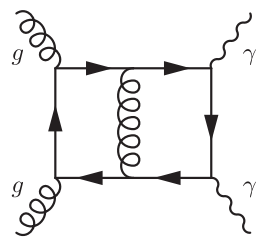

(d)

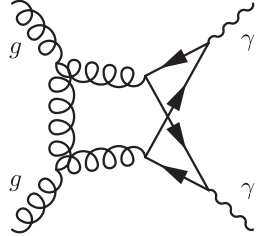

(e)

FIG. 1. Representative Feynman diagrams for $g g \rightarrow \gamma \gamma$ at NLO: the Born (a), real corrections (b,c), and virtual corrections (d,e). In our computation photons couple only to the quarks running in the closed loop, i.e., 5 massless quarks $u, d, c, s, b$ and the top quark $t$.

top mass and could provide a handle on a top quark mass that is free from the usual hadronic systematic uncertainties. In the ultra-high energy limit the top quark contribution can be estimated by treating it as a massless quark: naively summing over the electric charges, the inclusion of the top quark increases the gluon fusion contribution by $\left(\sum_{6 F} e_{q}^{2}\right)^{2} /\left(\sum_{5 F} e_{q}^{2}\right)^{2}-1 \approx 86 \%$.

The diphoton spectrum also provides a privileged observatory to search for new physics. While the resonant production of new physics particles decaying into diphoton can be searched with theory-independent side-band method, the nonresonant or interference cases, require a precise prediction of the SM contribution. Interference of the resonant contribution with the SM continuum, provides an important method to extract properties of the resonance, such as the width. The case of Higgs boson has been extensively investigated, see e.g., [18,19]. When new physics resonances are produced mostly via gluon fusion, such as for example scalars and spin-2 particles, the SM contribution can interfere determining nontrivial structures like peak-dip (or dip-peak) or just dip structures [20], depending on the couplings and properties of the resonance. As these new physics searches are particularly motivated above the top pair threshold, including the top-quark contribution is essential.

The computation of NLO corrections of the top-quark induced contributions, requires the knowledge of highly nontrivial two-loop amplitudes. While in the massless quark limit the corresponding amplitudes have been known for a long time [21], the computation of massive ones, is still a challenge. Analytical results have become available in closed form for some of the relevant Feynman integrals (planar) yet the full set is unknown. On the other hand, numerical methods have been introduced [22] that allow to perform this calculation. In this paper, we compute the complete NLO corrections to the gluon fusion channel $g g \rightarrow \gamma \gamma$, including the top-quark contribution for the first time.

\section{CAlCulation}

The cross section at NLO accuracy can be written as

$$
\mathrm{d} \sigma^{\mathrm{NLO}}=\mathrm{d} \sigma^{\mathrm{Born}}+\mathrm{d} \sigma^{\mathrm{V}}+\mathrm{d} \sigma^{\mathrm{R}}+\mathrm{d} \sigma^{\mathrm{C}},
$$

where $\mathrm{d} \sigma^{\text {Born }}$ is the leading order one-loop contribution, $\mathrm{d} \sigma^{V}$ denotes the virtual (two-loop) contributions, $\mathrm{d} \sigma^{R}$ is the real (one-loop, $2 \rightarrow 3$ contribution), and $\mathrm{d} \sigma^{C}$ represents the collinear singularity to absorbed into the parton distribution functions. The representative Feynman diagrams for the Born, virtual and real contributions are shown in Fig. 1. We define the gluon fusion process as the (gauge-invariant) set of contributions to diphoton production coming from amplitudes where the photons couple to gluons through a closed quark loop. At the cross section level we include terms which scale as $\left(\sum_{q} e_{q}^{2}\right)^{2}$ up to order $\alpha_{S}^{3}$. According to our definition, the two-loop contribution where a quarkantiquark pair annihilates into a virtual gluon pair which then couple to diphoton via a quark loop, can contribute to the gluon fusion process. However, in addition of not being enhanced by the gluon-gluon luminosity, this two-loop amplitude can only contribute to the $\left(\sum_{q} e_{q}^{2}\right)^{2}$ term via its square, which is $\mathcal{O}\left(\alpha_{S}^{4}\right)$ and therefore beyond the accuracy of our computation [23]. Each of the three terms at NLO are infrared/collinear divergent. Their sum, however, is free of infrared/collinear divergences. To handle this cancellation, we employ an in-house implementation of the dipole subtraction method [24], which introduces counterterms for each term $\mathrm{d} \sigma_{\text {fin }}^{i}=\mathrm{d} \sigma^{i}-\mathrm{d} \sigma_{\text {dipole }}^{i}$ with $i=\mathrm{V}, \mathrm{R}, \mathrm{C}$. The subtraction terms $\mathrm{d} \sigma_{\text {dipole }}^{i}$ are carefully chosen such that they cancel locally the infrared/collinear divergences of each term, and sum up to zero [24].

Once the subtraction method is in place, one is left with the calculation of the matrix elements for the virtual and real contributions. The latter corrections require the computation of one-loop five point amplitudes, which can be done automatically. In particular, the matrix element for $g g \rightarrow \gamma \gamma g$ subprocess, as well as $g q(\bar{q}) \rightarrow \gamma \gamma q(\bar{q})$ and $q \bar{q} \rightarrow \gamma \gamma g$ subprocesses are needed. To this aim, we adopt RECOLA2 [25] and MADGRAPH5_AMC@NLO [26], as well as analytical expression for the light-quark contributions [27-29].

We have implemented the light quark contribution from Ref. [21] in our code. We have then considered the calculation of the top-quark contribution. Two-loop diagrams have been generated by QGRAF [30], and processed by FORM [31,32], to generate corresponding amplitudes. They are fed into REDUZE [33] to perform the corresponding 
loop momentum redefinition and to classify them into 33 integral families according to the propagator structure. We then adopt a projection method to decompose the amplitudes into 10 independent tensor structures, reducing the computation into that of scalar integrals with irreducible numerators. Employing the C++ version of FIRE5 [34] with LITERED [35] to perform the integration-by-part reduction, we finally obtain the corresponding form factors as a linear combination of 1180 master integrals, distributed into the 33 integral families. We evaluate the master integrals family by family, not considering the relations among the master integrals of different families. The calculation of the master integrals is based on numerical integration of differential equations, with initial condition provided by an in-house implementation of sector decomposition method [36]. The numerical integration of differential equation is done with ODEINT [37]. Starting from the original initial conditions, several points in the physical region are pre-computed and results are stored. During the phase space integration, the closest point in the precomputed set is adopted as the new initial condition. The average time to evaluate the amplitude is around 1 second, with at least $\mathcal{O}\left(10^{-9}\right)$ precision at the master integral level. The one-loop amplitude up to $\mathcal{O}\left(\epsilon^{2}\right)$ order is computed within the same method. We refer the reader to [22] for more details on our method and its extensive validation. Here we stress that, whenever available, we have compared the numerical value of the master integrals with those in the literature [38-40], and found excellent agreement.

We renormalize $\alpha_{S}$ in the $\overline{\mathrm{MS}}$ scheme with five flavors. The top-quark mass is renormalized on shell. We have checked that UV divergences are cancelled by the corresponding counterterms, and IR and collinear divergences cancel with the dipole subtraction terms. We have also checked that our implementation for the massless contribution at NLO agrees with that of MCFM [15,41]. To monitor the numerical accuracy during the evaluation, we exploit the $t \leftrightarrow u$ symmetry and calculate two independent yet equivalent values of the integral in each point of the phase space. With the uncertainties estimated through adopting different initial conditions, as well as exchanging $t$ and $u$, we conclude that the uncertainty arising from the numerical evaluation is smaller than $0.4 \%$ times LO contribution, at both inclusive and differential level.

\section{RESULTS}

We adopt the following input parameters $\alpha(0)=$ $1 / 137.035999139, m_{t}=173.0 \mathrm{GeV}, \Gamma_{t}=1.41 \mathrm{GeV}$ [42]. In order to regulate Coulomb divergences in the top pair threshold region, we include the top quark width. We adopt the complex mass scheme, e.g., the top quark mass is replaced by the complex quantity $\mu_{t}=\sqrt{m_{t}^{2}-i m_{t} \Gamma_{t}}$ everywhere. The renormalization scale and factorization scale are set to $\mu_{R}=\mu_{F}=m(\gamma \gamma) / 2$, and we vary them by a factor of two around the central scale to assess scale

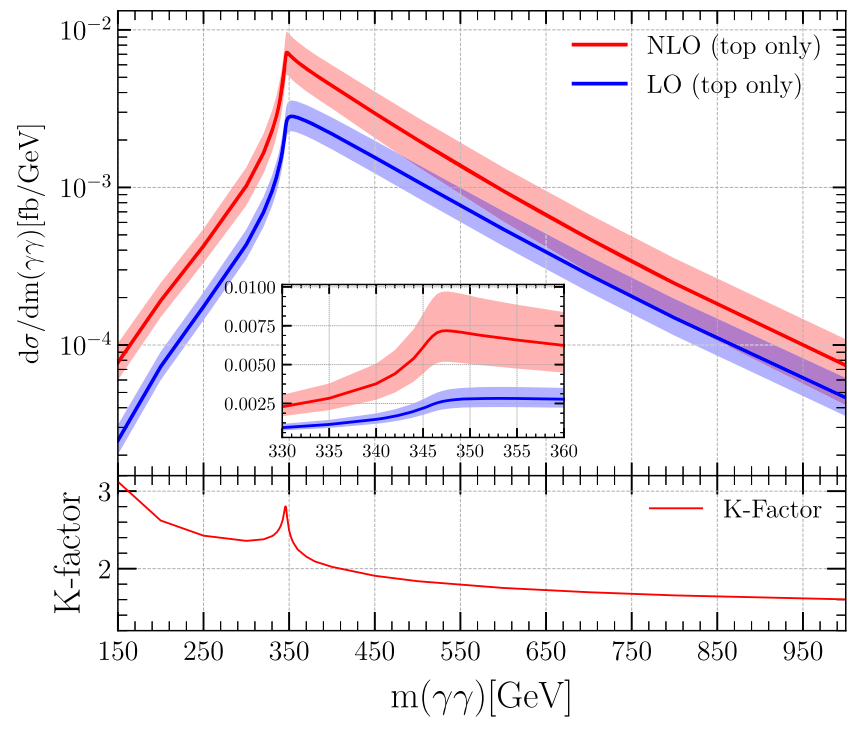

FIG. 2. The differential cross section in $m(\gamma \gamma)$ is shown for $g g \rightarrow \gamma \gamma$ considering only the top quark in the loop, with the bands indicating the scale uncertainties.

uncertainties. We choose PDF4LHC15_nlo_100_pdfas [43] and the corresponding $\alpha_{S}$ for both the LO and the NLO results, and the results are presented for $13 \mathrm{TeV}$ LHC. We apply the following cuts $p_{T}\left(\gamma_{1}\right)>40 \mathrm{GeV}$, $p_{T}\left(\gamma_{2}\right)>25 \mathrm{GeV},|\eta(\gamma)|<2.5$. No photon isolation is applied.

In Fig. 2, we show the differential cross section for the case where only the top-quark contribution is taken into account, at LO and NLO, as well as corresponding scale uncertainties. Both LO and NLO cross sections peak around the top-quark pair threshold. The NLO corrections lead to a large $K$-factor $\left(K^{\mathrm{NLO}}=\sigma^{\mathrm{NLO}} / \sigma^{\mathrm{LO}}\right)$, especially in the low invariant mass region. Even when the invariant mass $m(\gamma \gamma)$ is low, the total center of mass energy in the real correction can be above the top pair threshold, and thus the top-quark loop can get resolved, leading to such enhancement. Furthermore, the photon $p_{T}$ cuts enhance the real corrections since at LO photons are back-to-back and therefore have both $p_{T}>40 \mathrm{GeV}$, while at NLO the second photon can be softer. As $m(\gamma \gamma)$ increases, the $K$-factor decreases, reaching a local maximum of value around 2.8 at the top-quark pair threshold. In such a region, the top quarks in the loop are produced on shell and almost at rest, and can exchange a Coulomb gluon leading to an enhancement (tamed by the top-quark width). Such corrections are well known and universal. They can be resummed by employing bound state techniques, see e.g., [44], though we present only fixed-order results here.

For a better view of the top-quark contribution, in Fig. 3 we show the differential cross section close to the top pair threshold region. Here, the top-quark and the five massless quarks contributions have a different phase, leading to a destructive interference that decreases the cross section. 


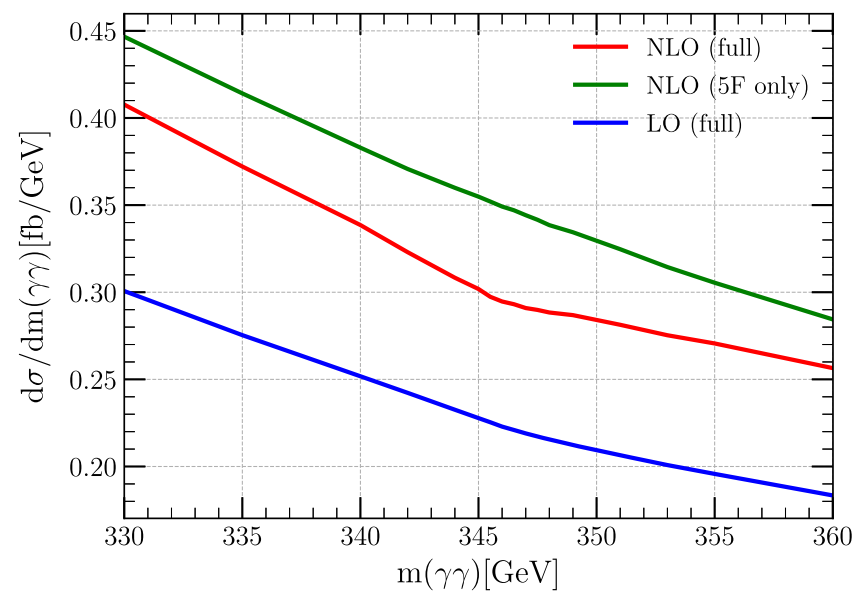

FIG. 3. The differential cross section in $m(\gamma \gamma)$ before and after the inclusion of the top-quark contribution in $g g \rightarrow \gamma \gamma$ is shown for the top pair threshold region.

As already mentioned, the exchange of a Coulomb gluon leads to large NLO corrections at the top-quark pair threshold. Thus, the destructive interference decreases the cross section further and the change of slope below and above twice the top mass is more visible at NLO than at LO. Our results provide a key ingredient for improving the resummed predictions in the threshold region and also reinforce the hope that such slope change could be exploited to extract a short-distance (potential) mass for the top quark [44]. Further studies will be needed to carefully assess the expected precision reachable at the LHC and at future colliders.

In the top inset of Fig. 4, we show the differential cross section at LO and NLO for the full result, i.e., including five massless quarks along with the top quark (full) and the NLO result for the massless quarks only (5F only) averaged over $50 \mathrm{GeV}$ wide bins. In the middle inset, we plot the $K$-factor $\left(K^{\mathrm{NLO}}=\sigma^{\mathrm{NLO}} / \sigma^{\mathrm{LO}}\right)$, which shows that NLO corrections for the top quark contribution in the high invariant mass regions are more important than those for the light quarks. Moreover, we plot the ratio between the "full" and "5F only" contributions at LO and NLO, in the lower inset. It clearly shows that the effect of the top quark

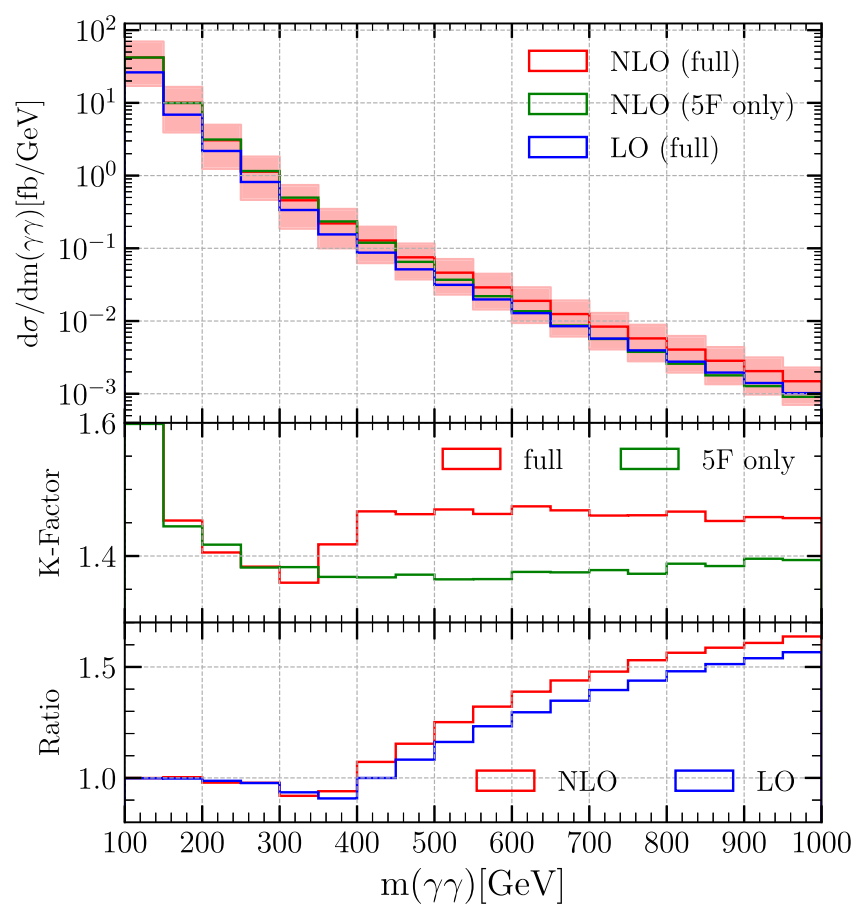

FIG. 4. The differential cross section in $m(\gamma \gamma)$ for $g g \rightarrow \gamma \gamma$ is shown in the cases of light quarks only and the full result (including the top quark). The band indicates the scale uncertainties for "NLO (full)". The corresponding $K$-factor as well as the ratio between full and $5 \mathrm{~F}$ are shown.

mass is negligible in the low energy region both at LO and NLO as the top-quark contribution is parametrically suppressed as $\mathcal{O}\left(s^{2} / m_{t}^{4}\right)$. As the energy increases toward the top-quark pair threshold, the inclusion of the top-quark contribution leads to destructive interference, thus decreases the cross section. This behaviour is not affected by NLO corrections. However, as evident from the ratio as well as the $K$-factor plots, above threshold NLO corrections become large. In the region $400 \mathrm{GeV}<m(\gamma \gamma)<1000 \mathrm{GeV}$, the full calculation yields a larger $K$-factor than the corresponding one obtained with massless quarks only. Reweighting the full LO contribution with the $K$-factor obtained from the lightquark contribution would therefore underestimate the full results by $5 \%-10 \%$. Starting at about $400 \mathrm{GeV}$, the top-quark

TABLE I. The differential cross section of $g g \rightarrow \gamma \gamma$ for various contribution at LO and NLO are shown for different values of diphoton invariant mass $m(\gamma \gamma)$. "5F only" means only including the five massless quarks, "top only" means only including the top quark, "interference" means only the interference term between the light quarks and the top quark, and "full" means all the above contributions. The cross section for $q \bar{q} \rightarrow \gamma \gamma$ is also shown for comparison.

\begin{tabular}{|c|c|c|c|c|c|c|c|c|c|}
\hline \multirow{2}{*}{$\frac{\mathrm{fb} / \mathrm{GeV}}{m(\gamma \gamma)[\mathrm{GeV}]}$} & \multirow[t]{2}{*}{$q \bar{q} \rightarrow \gamma \gamma, \mathrm{LO}$} & \multicolumn{4}{|c|}{$g g \rightarrow \gamma \gamma, \mathrm{LO}$} & \multicolumn{4}{|c|}{$g g \rightarrow \gamma \gamma$, NLO } \\
\hline & & Full & $5 \mathrm{~F}$ only & Top only & Interference & Full & $5 \mathrm{~F}$ only & Top only & Interference \\
\hline 125 & 47.1 & $24.26(1)$ & $100.1 \%$ & $<0.01 \%$ & $-0.1 \%$ & $37.3(1)$ & $100.1 \%$ & $<0.01 \%$ & $-0.1 \%$ \\
\hline 400 & 1.04 & $0.11342(5)$ & $104.6 \%$ & $1.9 \%$ & $-6.5 \%$ & $0.1628(5)$ & $99.3 \%$ & $2.7 \%$ & $-2.0 \%$ \\
\hline 500 & 0.427 & $0.03951(7)$ & $88.7 \%$ & $2.8 \%$ & $8.6 \%$ & $0.0582(2)$ & $82.7 \%$ & $3.5 \%$ & $13.9 \%$ \\
\hline 1000 & 0.0210 & $8.721(8) \times 10^{-4}$ & $63.2 \%$ & $5.3 \%$ & $31.5 \%$ & $1.266(2) \times 10^{-3}$ & $60.5 \%$ & $5.8 \%$ & $33.6 \%$ \\
\hline
\end{tabular}


contribution increases the cross section, and since the NLO corrections for the top-quark-only case are more important than the light-quark case, the full result displays a larger $K$ factor. As a consequence, at NLO the ratio between the full and $5 \mathrm{~F}$ only results is larger, slowly approaching the predictions for the $6 \mathrm{~F}$ (massless) calculation $(\approx 1.86)$.

In Table I, we provide benchmark values for the differential cross section. As discussed before, in the low invariant mass region the top-quark contribution is tiny. For example, at the Higgs mass region $m(\gamma \gamma)=m_{H}=125 \mathrm{GeV}$, it is around $-0.1 \%$. Going above top-quark pair threshold the top-quark contribution decreases the cross section at LO, but the NLO cross section is almost unchanged. Far above threshold, the interference turns to be constructive, and very slowly approaching the $6 \mathrm{~F}$ (massless) limit.

\section{CONCLUSIONS}

In this paper, we have presented the first complete computation of the NLO corrections to $g g \rightarrow \gamma \gamma$ in the standard model, including both light-quarks and top-quark contributions. We have studied the top-quark effects in the total cross section and differentially, focusing on the invariant mass spectrum of the photons. We find that the NLO corrections are important everywhere, but especially in the vicinity of the top-quark pair threshold, where indeed an enhancement is expected on general grounds. A remarkable feature of the NLO spectrum is that the change of slope at the $t \bar{t}$ threshold becomes much more evident. Our calculation paves the way to improving the treatment of the threshold region at NLO including (pseudo-) bound state effects, with the goal to extract a short-distance topquark mass, and to include background-signal interference effects at NLO accuracy in the production of new physics heavy scalar resonances decaying to diphoton final states.

\section{ACKNOWLEDGMENTS}

We thank Matteo Becchetti and Roberto Bonciani for providing us results of two-loop integrals for cross checks. We thank Claude Duhr and Pierpaolo Mastrolia for their advise. We are grateful to John Campbell for his help in comparing our code to the massless NLO computation in MCFM. This work has received funding by the Marie Skłodowska-Curie MCnetITN3 (grant agreement no. 722104) and by the F.R.S.-FNRS with the EOS-be.h Project No. 30820817. The work of M. K. M. is funded by the UniPD STARS Grant 2017 "Diagrammalgebra". Computational resources have been provided by the CISM at UCLouvain and the CÉCI funded by the F. R. S.-FNRS under convention 2.5020.11.
[1] T. Aaltonen et al. (CDF Collaboration), Measurement of the Cross Section for Prompt Isolated Diphoton Production Using the Full CDF Run II Data Sample, Phys. Rev. Lett. 110, 101801 (2013).

[2] V. M. Abazov et al. (D0 Collaboration), Measurement of the differential cross sections for isolated direct photon pair production in $p \bar{p}$ collisions at $\sqrt{s}=1.96 \mathrm{TeV}$, Phys. Lett. B 725, 6 (2013).

[3] S. Chatrchyan et al. (CMS Collaboration), Measurement of the production cross section for Pairs of isolated photons in $p p$ collisions at $\sqrt{s}=7 \mathrm{TeV}, \mathrm{J}$. High Energy Phys. 01 (2012) 133.

[4] S. Chatrchyan et al. (CMS Collaboration), Measurement of differential cross sections for the production of a pair of isolated photons in pp collisions at $\sqrt{s}=7 \mathrm{TeV}$, Eur. Phys. J. C 74, 3129 (2014).

[5] G. Aad et al. (ATLAS Collaboration), Observation of a new particle in the search for the Standard Model Higgs boson with the ATLAS detector at the LHC, Phys. Lett. B 716, 1 (2012).

[6] S. Chatrchyan et al. (CMS Collaboration), Observation of a new boson at a mass of $125 \mathrm{GeV}$ with the CMS experiment at the LHC, Phys. Lett. B 716, 30 (2012).

[7] V. Khachatryan et al. (CMS Collaboration), Search for highmass diphoton resonances in protonproton collisions at
$13 \mathrm{TeV}$ and combination with $8 \mathrm{TeV}$ search, Phys. Lett. B 767, 147 (2017).

[8] M. Aaboud et al. (ATLAS Collaboration), Search for new phenomena in high-mass diphoton final states using $37 \mathrm{fb}^{-1}$ of proton-proton collisions collected at $\sqrt{\mathrm{s}}=$ $13 \mathrm{TeV}$ with the ATLAS detector, Phys. Lett. B 775, 105 (2017).

[9] I. Antoniadis, A. Arvanitaki, S. Dimopoulos, and A. Giveon, Phenomenology of TeV Little String Theory from Holography, Phys. Rev. Lett. 108, 081602 (2012).

[10] M. Baryakhtar, Graviton phenomenology of linear dilaton geometries, Phys. Rev. D 85, 125019 (2012).

[11] P. Cox and T. Gherghetta, Radion dynamics and phenomenology in the linear dilaton model, J. High Energy Phys. 05 (2012) 149.

[12] G. F. Giudice, Y. Kats, M. McCullough, R. Torre, and A. Urbano, Clockwork/linear dilaton: Structure and phenomenology, J. High Energy Phys. 06 (2018) 009.

[13] T. Binoth, J. P. Guillet, E. Pilon, and M. Werlen, A full nextto-leading order study of direct photon pair production in hadronic collisions, Eur. Phys. J. C 16, 311 (2000).

[14] S. Catani, L. Cieri, D. de Florian, G. Ferrera, and M. Grazzini, Diphoton Production at Hadron Colliders: A Fully-Differential QCD Calculation at NNLO, Phys. Rev. Lett. 108, 072001 (2012); 117, 089901(E) (2016). 
[15] J. M. Campbell, R. K. Ellis, Y. Li, and C. Williams, Predictions for diphoton production at the LHC through NNLO in QCD, J. High Energy Phys. 07 (2016) 148.

[16] M. Grazzini, S. Kallweit, and M. Wiesemann, Fully differential NNLO computations with MATRIX, Eur. Phys. J. C 78, 537 (2018).

[17] Z. Bern, L. J. Dixon, and C. Schmidt, Isolating a light Higgs boson from the diphoton background at the CERN LHC, Phys. Rev. D 66, 074018 (2002).

[18] L. J. Dixon and Y. Li, Bounding the Higgs Boson Width Through Interferometry, Phys. Rev. Lett. 111, 111802 (2013).

[19] J. Campbell, M. Carena, R. Harnik, and Z. Liu, Interference in the $g g \rightarrow h \rightarrow \gamma \gamma$ On-Shell Rate and the Higgs Boson Total Width, Phys. Rev. Lett. 119, 181801 (2017); 119, 199901(A) (2017).

[20] D. Chway, R. Dermíšek, T. H. Jung, and H. D. Kim, Gluons to Diphotons via New Particles with Half the Signals Invariant Mass, Phys. Rev. Lett. 117, 061801 (2016).

[21] Z. Bern, A. De Freitas, and L. J. Dixon, Two loop amplitudes for gluon fusion into two photons, J. High Energy Phys. 09 (2001) 037.

[22] M. K. Mandal and X. Zhao, Evaluating multi-loop Feynman integrals numerically through differential equations, J. High Energy Phys. 03 (2019) 190.

[23] The interference with the tree-level $q \bar{q} \rightarrow \gamma \gamma$ amplitude is finite and scales as $e_{q}^{2}\left(\sum_{q} e_{q}^{2}\right)$. In the literature, these terms (together with the one-loop/tree-level real $2 \rightarrow 3$ interference counterparts which are also finite) have been included in the NNLO $q \bar{q} \rightarrow \gamma \gamma$ computation, evaluating them with massless quarks in the loops. Their contributions to the cross section has been found marginal.

[24] S. Catani and M. H. Seymour, A general algorithm for calculating jet cross-sections in NLO QCD, Nucl. Phys. B485, 291 (1997); Erratum, Nucl. Phys. B510, 503(E) (1998).

[25] A. Denner, J.-N. Lang, and S. Uccirati, Recola2: Recursive computation of one-loop amplitudes 2, Comput. Phys. Commun. 224, 346 (2018).

[26] J. Alwall, R. Frederix, S. Frixione, V. Hirschi, F. Maltoni, O. Mattelaer, H. S. Shao, T. Stelzer, P. Torrielli, and M. Zaro, The automated computation of tree-level and next-toleading order differential cross sections, and their matching to parton shower simulations, J. High Energy Phys. 07 (2014) 079.

[27] Z. Bern, L. J. Dixon, and D. A. Kosower, One Loop Corrections to Five Gluon Amplitudes, Phys. Rev. Lett. 70, 2677 (1993).
[28] D. de Florian and Z. Kunszt, Two photons plus jet at LHC: The NNLO contribution from the gg initiated process, Phys. Lett. B 460, 184 (1999).

[29] C. Balazs, P. M. Nadolsky, C. Schmidt, and C. P. Yuan, Diphoton background to Higgs boson production at the LHC with soft gluon effects, Phys. Lett. B 489, 157 (2000).

[30] P. Nogueira, Automatic Feynman graph generation, J. Comput. Phys. 105, 279 (1993).

[31] J. A. M. Vermaseren, New features of FORM, arXiv: math-ph/0010025.

[32] J. Kuipers, T. Ueda, J. A. M. Vermaseren, and J. Vollinga, FORM version 4.0, Comput. Phys. Commun. 184, 1453 (2013).

[33] A. von Manteuffel and C. Studerus, Reduze 2-Distributed Feynman Integral REduction, arXiv:1201.4330.

[34] A. V. Smirnov, FIRE5: A C++ implementation of Feynman Integral REduction, Comput. Phys. Commun. 189, 182 (2015).

[35] R. N. Lee, Presenting LiteRed: A tool for the loop InTEgrals REDuction, arXiv:1212.2685; LiteRed 1.4: A powerful tool for reduction of multiloop integrals, J. Phys. Conf. Ser. 523, 012059 (2014).

[36] T. Binoth and G. Heinrich, An automatized algorithm to compute infrared divergent multiloop integrals, Nucl. Phys. B585, 741 (2000).

[37] K. Ahnert and M. Mulansky, Odeint—Solving ordinary differential equations in $\mathrm{C}++$, AIP Conf. Ser. 1389, 1586 (2011).

[38] S. Caron-Huot and J. M. Henn, Iterative structure of finite loop integrals, J. High Energy Phys. 06 (2014) 114.

[39] A. von Manteuffel and L. Tancredi, A non-planar twoloop three-point function beyond multiple polylogarithms, J. High Energy Phys. 06 (2017) 127.

[40] M. Becchetti and R. Bonciani, Two-loop Master integrals for the planar QCD massive corrections to di-photon and dijet hadro-production, J. High Energy Phys. 01 (2018) 048.

[41] A comparison with our results has allowed to identify two bugs in the MCFM v8.0 implementation of minor numerical relevance, which have been corrected in MCFM v8.2. In addition the change of scheme to " $\mathrm{tH}-\mathrm{V}$ " needs to be applied to obtain full agreement with our results. This will be included in the forthcoming release of MCFM.

[42] M. Tanabashi et al. (Particle Data Group), Review of particle physics, Phys. Rev. D 98, 030001 (2018).

[43] J. Butterworth et al., PDF4LHC recommendations for LHC Run II, J. Phys. G 43, 023001 (2016).

[44] S. Kawabata and H. Yokoya, Top-quark mass from the diphoton mass spectrum, Eur. Phys. J. C 77, 323 (2017). 